\title{
De Novo Peptide Sequencing \\ Using Exhaustive Enumeration of Peptide Composition
}

\author{
Matthew T. Olson \\ Laboratory of Cellular and Molecular Biophysics, NICHD, NIH, Bethesda, Maryland, USA
}

Jonathan A. Epstein

Unit of Biologic Computation, OSD, NICHD, NIH, Bethesda, Maryland, USA

\author{
Alfred L. Yergey \\ Laboratory of Cellular and Molecular Biophysics, NICHD, NIH, Bethesda, Maryland, USA
}

We introduce the use of a peptide composition lookup table indexed by residual mass and number of amino acids for de novo sequencing of polypeptides. Polypeptides of 1600 Daltons (Da) or more can be sequenced effectively through exhaustive compositional analysis of MS/MS spectra obtained by unimolecular decomposition (without CID) in a MALDI TOF/ TOF despite a fragment mass accuracy of $50 \mathrm{mDa}$. Peaks are referenced against the lookup table to obtain a complete profile of amino acid combinations, and combinations are assembled into series of increasing length. Concatenating the differences between successive entries in compositional series yields peptide sequences that can be scored and ranked according to signal intensity. While the current work involves measurements acquired on MALDI TOFTOF, such general treatment of the data anticipates extension to other types of mass analyzers. (J Am Soc Mass Spectrom 2006, 17, 1041-1049) (C) 2006 American Society for Mass Spectrometry

W hile protein identification most commonly relies on established database searching routines [1-4], examples persist of demand for fast and reliable peptide sequencing due to incomplete or inadequate databases [5-9]. De novo peptide sequencing algorithms also abound [5, 10-17], but objective assessments of de novo peptide sequencing capabilities with unknowns [18] indicate that much work remains.

One recently described de novo sequencing method has taken an approach that is wholly different from others reported to date [15]. It employs logical processing of all amino acid combinations from the parent ion and prominent fragments to obtain several relevant amino acid combinations, which are permuted into sequences; the presence of other peaks guides the selection of a final sequence. This method presents a straightforward and desirable means for sequence validation and for obtaining sequences of peptides independently of how well they fragment. However, the resolution and mass accuracy specifications for this

Published online June 2, 2006

Address reprint requests to Dr. A. L. Yergey, NIH, Building 10, Room 9D52, Bethesda, MD 20892, USA. E-mail: aly@helix.nih.gov method rely completely on the desirable performance characteristics of Fourier transform mass spectrometry (FTMS).

The use of exhaustive peptide-relevant elemental combinations, as Spengler [15] has done, represents one particular understated advantage: it allows the inherent distinction of the correct ion type for use in sequence analysis. Thus, the generation of a sequence de novo is not misguided by neutral losses and/or high-energy fragmentation $\left(\mathrm{x}^{-}, \mathrm{w}_{-}^{-}, \mathrm{c}^{-}\right.$, or $\left.\mathrm{d}-\right)$ ions. Nearly all previously published de novo peptide sequencing methods [10-16] incorporate neutral loss, high-energy fragmentation, and a-type ions into the scoring and selection of the ultimate sequence. Extensive variability in any solution generated by them appears to stem from the implementation of this feature; a robust comparison of the advantages and shortcomings in these various scoring schemes, involving fragmentation simulations [16, 19], arbitrary values [13, 15], empirical assignment functions [14], or a combination, seems a worthwhile venture, especially in view of peptide and instrumental variability. While the importance of these nonsequence ions to the reinforcement of sequence validation cannot be underestimated, their highly variable frequency and intensity present significant obstacles to their accurate 
characterization. This may be the cause of the undesirable sequence shunting observed in algorithms that rely on neutral loss and high-energy fragmentation ions excessively.

Tandem TOF spectra obtained via unimolecular decompositions, i.e., without CID, contain more extensive-generally complete-fragmentations. This superior fragmentation carries a penalty of somewhat decreased mass accuracy in the fragment ions, compared with FTMS spectra, as a consequence of energy distributions within the analyzers being only partially resolved; in this paper the mass accuracy of TOF-TOF fragment spectra is assumed to be $50 \mathrm{mDa}$. While it is unclear what would be the effect of such large mass errors on the only published use of amino acid combinations for de novo sequencing [15], previous strategies for sequencing with TOF-TOF data $[5,14]$ do not fully exploit the potential of using mass accuracy data. This may partially explain some of their erroneous results. The approach described here utilizes a reasonable mass accuracy for unimolecular decomposition fragments and the straightforward compositional analysis allowed by LIPCUT (length incremented peptide composition lookup table) to gain highly reliable sequence information from TOF-TOF data.

\section{Experimental}

\section{Materials}

Acetonitrile (ACN HPLC grade), TFA, reagent grade dihydrogen ammonium phosphate (AmPhos), and a-cyano-4-hydroxycinnamic acid (HCCA) were obtained from Aldrich Chemical Co. (Milwaukee, WI); HCCA was recrystallized before use. The b-galactosidase digest and three peptides des-Arg1-Bradykinen, angiotensin 1, and glu-fibrinogen peptides were obtained from Applied Biosystems Inc. (Framingham, MA); the three individual peptides are part of a mixture of calibration standards, ABI4700 Calibration Mixture. BSA was obtained from Sigma Chemical Co. (St. Louis, $\mathrm{MO})$, digested in solution under standard conditions using sequencing grade modified porcine trypsin (Promega, Madison, WI). Water for general use was generated using a Hydro Services (Rockville, MD) deionizing purification system.

\section{Mass Spectrometry}

Samples were formed by applying $0.3 \mathrm{uL}$ of one of the three peptide mixtures to an Applied Biosystems 192 well OptiPlate disposable sample stage; peptide concentrations were all adjusted to be $1 \mathrm{pmol} / \mathrm{uL}$. An equal volume of matrix solution was applied to each peptide mixture before it had dried; the matrix solution was 5 $\mathrm{mg} / \mathrm{mL}$ HCCA dissolved in $900 \mathrm{uL}$ of $1: 1:: 0.1 \%$ TFA: ACN to which $100 \mathrm{uL}$ of $0.1 \mathrm{M}$ solution of AmPhos was added.

MALDI MS and MS/MS spectra were obtained in a
Table 1. Parameters for LIPCUT generation

\begin{tabular}{cclcc}
\hline Ratio & $\begin{array}{c}\text { Min } \\
\text { value }\end{array}$ & \multicolumn{1}{c}{$\begin{array}{c}\text { Min } \\
\text { value AAs }\end{array}$} & $\begin{array}{c}\text { Max } \\
\text { value }\end{array}$ & $\begin{array}{c}\text { Max } \\
\text { value AAs }\end{array}$ \\
\hline \hline $\mathrm{C} / \mathrm{C}$ & 1.0 & All & 1.0 & All \\
$\mathrm{H} / \mathrm{C}$ & .91 & $\mathrm{~W}$ & 2.0 & $\mathrm{~K}, \mathrm{R}$ \\
$\mathrm{N} / \mathrm{C}$ & .11 & $\mathrm{Y}, \mathrm{F}$ & .67 & $\mathrm{R}$ \\
$\mathrm{O} / \mathrm{C}$ & .09 & W & .75 & $\mathrm{D}$ \\
$\mathrm{S} / \mathrm{C}$ & 0 & All except M,C & .33 & $\mathrm{C}$ \\
\hline
\end{tabular}

The minimum and maximum values for the ratios of $\mathrm{C}, \mathrm{H}, \mathrm{N}, \mathrm{O}$ and $\mathrm{S}$ against $\mathrm{C}$ for amino acid residues as well as the amino acid where these ratios are found

semi-automated fashion using an Applied Biosystems 4700 Proteomics Analyzer with TOF/TOF ion optics (Applied Biosystems, Inc); in MS/MS mode the instrument was always operated with the collision gas off. A diode pumped Nd:YAG laser with a 600 ps pulse length was used. The instrument has been described in detail elsewhere $[5,20]$. The instrument was controlled by ABI 4700 Series Explorer (version 3.0). All calibrations were performed only after allowing instrument voltages to stabilize for at least $60 \mathrm{~min}$ in the operation mode. The sample plate calibration file was updated for each plate using five spots of the ABI 4700 calibration mixture. MS mode default calibration was updated daily using a calibration mixture spot in the center of the plate. MS/MS default calibration was updated based on successfully performing an internal calibration using the y-series fragment ions of Glu-fibrinogen peptide, $\mathrm{m} / \mathrm{z}$ 1570.677; criteria for successful MS/MS internal calibration included maximum outlier error no greater than $0.035 \mathrm{Da}$ and that at least 12 fragment ion masses were found.

Spectra were obtained for each sample using 500 laser shots in MS mode and 3500 shots in MS/MS; up to five MS/MS spectra were obtained from each spot. Tandem mass acquired by accelerating precursor ions to $8 \mathrm{keV}$, selecting them with the timing gate (TIS) set to a resolution of 50 and then accelerating fragment ions to $14 \mathrm{keV}$ before entering the reflector.

\section{Peptide Composition Lookup Table}

The basis of the entire approach taken here is the generation of the LIPCUT database that contains all peptide residue mass combinations for masses less than $2 \mathrm{kDa}$. It should be noted at this point that the LIPCUT database consists of a nonredundant listing of residue masses, i.e., peptide masses absent a mole of water; as will be shown below, this is a more general approach than simply listing peptide masses because of the greater flexibility provided in generating sequences. The table was generated by two independent methods, which were then used for cross validation: simple recursion and elemental combination. The isobars leucine and isoleucine were treated as the same residue and given the symbol $\mathrm{X}$ for all calculations. Recursion on the 19 residues was performed using alphabetically 
sorted amino acid combinations. To generate the table using elemental combination, minimum and maximum ratios relative to carbon were calculated for the five elements found in peptides, $\mathrm{C}, \mathrm{H}, \mathrm{N}, \mathrm{O}$, and S. Table 1 shows the bounds for ratios. The monoisotopic mass of a peptide is the sum of contributions from individual elements:

$$
\text { mass }=\mathrm{M}_{\mathrm{C}} \mathrm{N}_{\mathrm{C}}+\mathrm{M}_{\mathrm{H}} \mathrm{N}_{\mathrm{H}}+\mathrm{M}_{\mathrm{N}} \mathrm{N}_{\mathrm{N}}+\mathrm{M}_{\mathrm{O}} \mathrm{N}_{\mathrm{O}}+\mathrm{M}_{\mathrm{S}} \mathrm{N}_{\mathrm{S}}
$$

where $\mathrm{N}_{\mathrm{C}}, \mathrm{N}_{\mathrm{H}}, \mathrm{N}_{\mathrm{N}}, \mathrm{N}_{\mathrm{O}}$, and $\mathrm{N}_{\mathrm{S}}$ are the respective numbers of moles of $\mathrm{C}, \mathrm{H}, \mathrm{N}, \mathrm{O}$, and $\mathrm{S}$, and $\mathrm{M}_{\mathrm{C}}, \mathrm{M}_{\mathrm{H}}, \mathrm{M}_{\mathrm{N}}$, $\mathrm{M}_{\mathrm{O}}$, and $\mathrm{M}_{\mathrm{S}}$ are monoisotopic masses for $\mathrm{C}, \mathrm{H}, \mathrm{N}, \mathrm{O}$, and S. Using $\mathrm{H}$ and the maximum $\mathrm{H} / \mathrm{C}$ ratio as an example in eq 2, the maximum moles of hydrogen for any mass can be expressed in terms of the minimum moles of carbon.

$$
\mathrm{N}_{\max \mathrm{H}}=\mathrm{N}_{\min \mathrm{C}} \mathrm{R}_{\max \mathrm{H} / \mathrm{C}}
$$

Thus, the minimum number of carbons in a peptide for a given mass is known by a simple linear equation utilizing the maximum element versus carbon ratios in eq 3.

$$
\begin{array}{r}
N_{\text {min } C}=\frac{\text { mass }}{M_{C}+M_{H} R_{\max H / C}+M_{N} R_{\max N C}} \\
+M_{O} R_{\max O C}+M_{S} R_{\max S C}
\end{array}
$$

The maximum number of carbons can be obtained by substituting the minimum element versus carbon ratios from Table 1 into eq 3 . The same method can be used for substituting the minimum and maximum amounts of any element in a peptide for a given mass. Determination of the amino acid combinations within those elemental bounds and within a specified mass window yields the amino acid combinations for the mass.

Several indexing schemes and formats were investigated with the goal of optimizing computational speed for high throughput lookup. The LIPCUT used in this paper is formatted as a Berkeley database [21]. The primary key of the database is the number of amino acids in the combination, and the single secondary key is the sum of amino acid residue monoisotopic mass of that combination carried to five decimal places. This configuration was chosen because it balances the demands for functionality as well as for size and portability. Sorting and indexing the LIPCUT two ways allows for a more efficient processing of information: the mass increments allow for easy lookup with few comparisons, and the length increments allow for a grouping of compositions in a way that economizes the linking of series. Generation of LIPCUT, which only needs to be done once, utilized the high-performance computational capabilities of the Biowulf PC/Linux cluster at the National Institutes of Health, Bethesda, MD [22]. LIPCUT is available upon request.

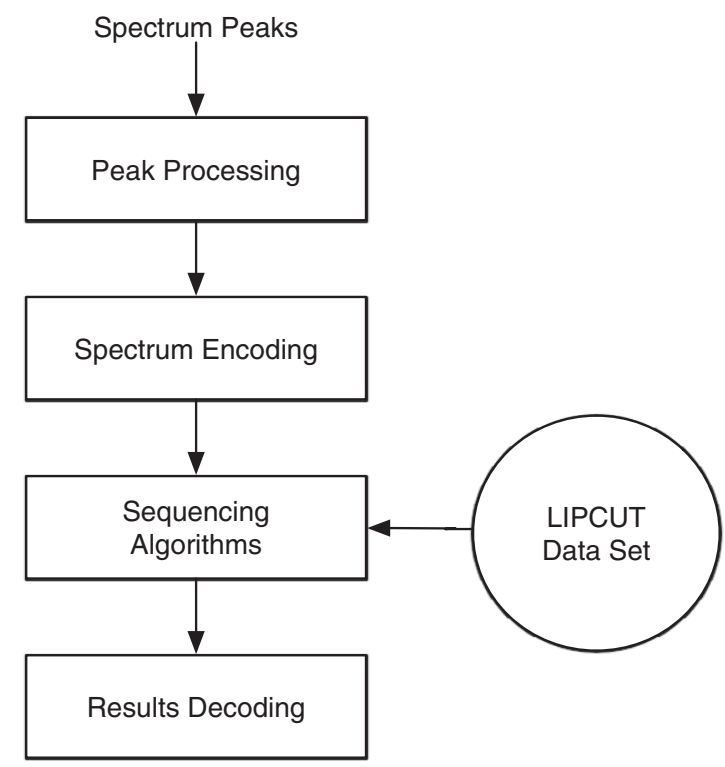

Scheme 1

\section{Development of a Sequence}

Scheme $\mathbf{1}$ is a flow chart of the process for development of a peptide sequence from an MS/MS spectrum. The first two steps are input functions that could be tailored to any type of fragmentation spectra; they are used to format the spectral data in a manner that an algorithm can employ LIPCUT. The final step is an output function that takes the results of the algorithm and arranges them in an interpretable fashion. The overall package of programs has been named MAARIAN (mass amino acid analysis by residue index for association of nodes).

\section{Peak Processing}

The ABI 4700 Series Explorer (version 3.0) software reports peak intensity as an integer multiple of signal versus noise $(\mathrm{S} / \mathrm{N})$. For each spectrum in these experiments, a peak list containing all peaks above a $\mathrm{S} / \mathrm{N}$ value of 3 was exported as a flat text file from the Explorer software. We found it necessary to implement a peak filtering step to reduce excessive noise in spectra with overall low intensities. A typical signal histogram for the spectra used in this work is shown in Figure 1. Peaks having S/N values with frequency greater than an arbitrary value of 10 were classified as intense. Low $\mathrm{S} / \mathrm{N}$ and high-frequency signals increase processing time [12] and generally present no helpful information for estimating sequence; they are filtered from a dataset for compositional analysis but saved for the use of elucidating other fragments. Due to the irregularity of noise distributions, it was found that signal filtration based solely on either frequency or $\mathrm{S} / \mathrm{N}$ occasionally removes useful signal. To preserve the fidelity of the peak list, our approach erred on the side of less filtration rather than more; all 
(a)

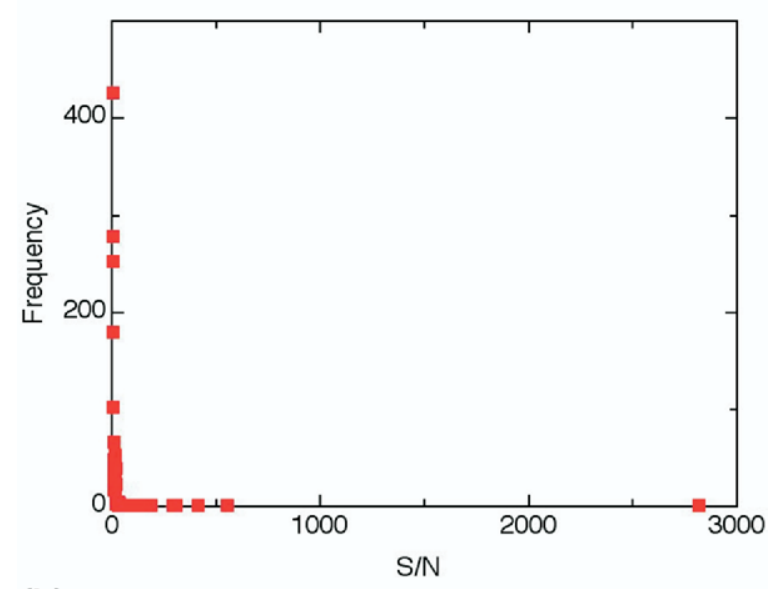

(b)

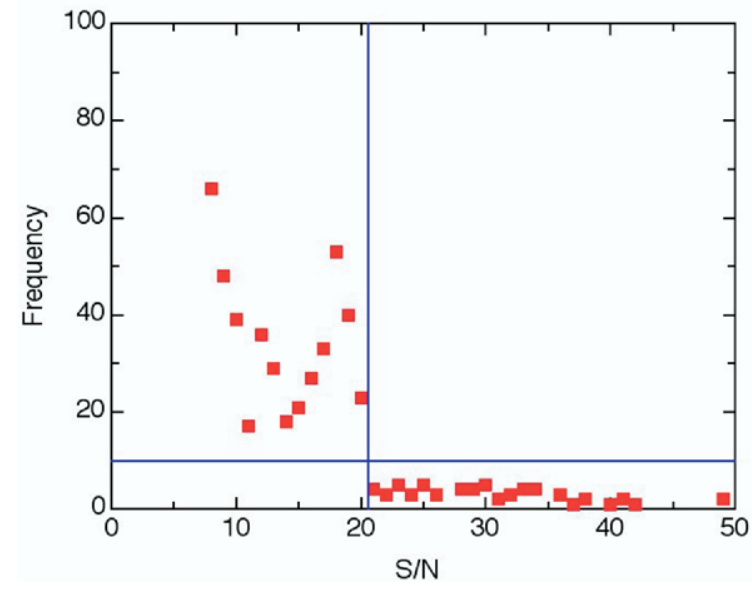

Figure 1. (a) $\mathrm{S} / \mathrm{N}$ histogram for all the signal in a spectrum of the tryptic BSA fragment 42-51 (XVNEXTEFAK). (b) Enlarged portion of the histogram; the horizontal line shows the arbitrary frequency cutoff; the minimum S/N with a frequency $<10$ occurs at S/N 21, so every peak with a $\mathrm{S} / \mathrm{N}>21$ (shown by the vertical line) is considered during compositional analysis

peaks with a $\mathrm{S} / \mathrm{N}$ greater than that having a frequency of 10 or less were used for analysis. Intensities of the filtered peaks were normalized to 1000 to clarify scoring.

Currently the S/N filter considers only monoisotopic peaks. Other components of the isotope cluster or any potential multiply charged ions, unlikely as they might be in MALDI, are ignored. Extension of this method to spectra generated by electrospray ionization will require an additional peak processing step to detect and deconvolute isotope clusters for multiply charged ions.

\section{Encoding the Spectrum}

Since LIPCUT is based on peptide residue masses, filtered spectra must be encoded appropriately to make use of them. At this point in the development of our approach, we treat all ions of a spectrum that pass the filter as either $y$ - or b-series ions recognizing that this assumptions is not likely to be entirely accurate. The encoding step treats each filtered peak both as a y- or b-series ion, by subtracting the monoisotopic molecular weight of $\mathrm{H}_{3} \mathrm{O}$ (19.01839 Da) from the measured mass in the first case, and by subtracting the mass of a hydrogen atom $(1.007825 \mathrm{Da})$ in the second. It is these "residual mass ions" that are referenced against LIPCUT in subsequent calculations. With this approach each ion in a spectrum will possibly, depending on its exact mass, lead to two different amino acid compositions, but care has been taken to include any given ion only once in a sequence, preferentially as a member of a y-series. The basis of the algorithms is that compositions inconsistent with a complete sequence are simply eliminated from further consideration. Peaks representing non-y or non-b ions simply go unassigned in the spectrum.

\section{De Novo Peptide Sequencing Algorithm}

A family of five algorithms that function either by chain extension or matching through some criterion of the spectrum and database have been developed to exploit the properties of LIPCUT. All of them are written as open source code and will be available upon request following the publication of a paper describing them in detail, along with a number of performance benchmarks [23]. The results presented herein have used one of the extension algorithms that assembles compositional series of increasing length from the exhaustive lists of ionic compositions found in the spectrum after referring to LIPCUT. It is similar to the stepwise approach [5] used earlier, but modified to operate with the exact masses and large numbers of amino acid compositions encountered. Additionally, contrary to previous uses of this approach, no initial assumptions are required before the sequence is generated. Growing compositional series need not reach the parent ion residual mass due to $\mathrm{b}$ complementation of $\mathrm{y}$ ions and vice versa. The algorithm can handle extensions to accommodate any number of missed fragmentations, but for this initial work, a single missed fragmentation (the addition of two amino acids) has been taken as the acceptable limit.

\section{Decoding Compositions}

The aforementioned sequencing algorithms generate series of $y$ - and b-type amino acid compositions from which complete amino acid sequences can be derived. In the simplest case, the entire sequence can be derived from a single ion type, and the series consists of amino acid compositions of ascending length. Decoding this case involves concatenating the amino acid differences between the compositions of ascending members of the series and arranging the differences in the appropriate order ( $\mathrm{N}$ - to C-terminal for $\mathrm{b}$-series, and $\mathrm{C}$ - to $\mathrm{N}$ terminal for $y$-series). While the $y$-series in unimolecular decompositions can be sufficient for the deduction of 
Table 2. Results Summary

\begin{tabular}{|c|c|c|c|c|}
\hline & Precursor & Expected seq & MAARIAN-LIPCUT seq ${ }^{a, b}$ & $\begin{array}{c}\text { Execution time } \\
(\mathrm{sec})^{\mathrm{c}}\end{array}$ \\
\hline \multicolumn{5}{|l|}{ b-Galactosidase } \\
\hline $232-238$ & 900.385 & FNDDFSR & (FN)DDFSR & 0.22 \\
\hline $433-440$ & 989.488 & WLPAMSER & WXPAMSER & 0.26 \\
\hline $954-962$ & 1083.522 & GDFQFNISR & {$[(\mathrm{DG}) \mid(\mathrm{AT})] \mathrm{FKFNXSR}$} & 3.09 \\
\hline $802-810$ & 1099.554 & IDPNAWVER & [(DX)|(EV)]PNAWVER & 3.27 \\
\hline $623-631$ & 1265.642 & HQQQFFQFR & HQQQFFKFR & 0.64 \\
\hline $944-953$ & 1299.623 & ELNYGPHKWR & EXNYGPHKWR & 1.43 \\
\hline $507-518$ & 1341.669 & VDEDQPFPAVPK & VDED[K|Q]PFPAV(KP) & 5.60 \\
\hline 168-169 & 1394.732 & LPSEFDLSAFLR & (PX)SEFDXSAFXR & 0.38 \\
\hline $16-27$ & 1428.687 & DWENPGVTQLNR & (DW)ENPGVTKXNR & 1.45 \\
\hline N-Terminal & 1446.841 & $\ldots$ LAVVLQR $^{\mathrm{d}}$ & (MT)XTDSXAVVXKR & 0.64 \\
\hline $963-974$ & 1507.696 & YSQOQLMETSHR & YSQQ(KX)METSHR & 0.22 \\
\hline \multicolumn{5}{|l|}{$B S A$} \\
\hline $161-167$ & 927.494 & YLYEIAR & YXYEXAR & 8.82 \\
\hline $66-75$ & 1163.631 & LVNELTEFAK & (VX)NEXTEFAK & 1.29 \\
\hline $25-34$ & 1193.603 & DTHKSEIAHR & DTHKSEXAHR & 0.46 \\
\hline $35-44$ & 1249.622 & FKDLGEEHFK & FKDXGEEH(FK) & 3.19 \\
\hline $402-412$ & 1305.717 & HLVDEPONLIK & HXVDEP[K|Q]NX(KX) & 0.78 \\
\hline $437-451$ & 1639.938 & KVPQVSTPLVEVSR & KVPQVS(PT)LVEVSR & 0.46 \\
\hline \multicolumn{5}{|l|}{ Misc Peptides } \\
\hline des-Arg1-Bradykinin & 904.460 & PPGFSPFR & PPGFSPFR & 1.91 \\
\hline Angiotensin 1 & 1296.685 & DRVYIHPFHL & DRVYXHPF(HX) & 16.57 \\
\hline Glu-Fibrino peptide & 1570.677 & EGVNDNEEGFFSAR & {$[(E G) \mid(A D)] V N D N E E G F F S A R$} & 0.87 \\
\hline Synthetic 1 & 1018.408 & SAQEPSQGNT & SAQEPS $\{E D R\}$ & 6.84 \\
\hline Synthetic 2 & 1166.416 & DNDEEEGPFD & DNDEEEGPFD & 5.02 \\
\hline
\end{tabular}

${ }^{a}$ In this table $X$ represents the isobaric amino acids Ile and Leu.

${ }^{b}$ Missed fragmentations are shown within parentheses and near-isobaric mass alternatives within square brackets. Incorrect sequences are within braces.

cAverage time using $2.5 \mathrm{GHz}$ Power Mac G5.

dTe published N-terminal sequence for this peptide is: MVASSLALAVVLQR

a complete sequence, the algorithms expand the series with either $y$ - or b-compositions.

\section{Sequence Scoring}

The score for the sequence is the sum of intensities for all the peaks, which have amino acid compositions that support the sequence. Attempts were made to incorporate a calculation of mass error into the sequence score, but no improvement was realized.

\section{Results and Discussion}

The results for determining the sequence of 22 standard peptides from three different sources are summarized in Table 2. Of these 22, seven are determined correctly with no ambiguities other than being unable to resolve the near isobars of $K$ and $Q$. A significant aspect of these results is that the b-galactosidase peptide at $\mathrm{m} / \mathrm{z}$ 1446.841 had a C-terminal portion consistent with a portion of the protein's amino terminal, but the sequence found was longer than that published for this peptide. We speculate that this recombinant form of the protein was synthesized by cloning a vector that employed a variant amino terminal. Of the other 15 peptides, 11 were determined to within a single missed fragmentation, shown by two residues within parenthe- ses, or two isobaric pairs within parentheses, one of which is correct, within square brackets. Of this set of 15,11 were clarified by the use of internal fragment ions to yield the correct sequences as discussed below. Only one peptide, synthetic 1 , a nontryptic-like synthetic molecule, yielded results that differed appreciably from the correct sequence at the C-terminus; the incorrect residues are shown in bold italic within braces; the portion of the sequence that was correct was ranked second, the only instance of missing a correct determination.

The execution time to produce these sequences is given in the last column of the table. All calculations were performed using a $2.5 \mathrm{GHz}$ Power Mac G5 running OS 10.4.5 and Perl 5.8.4. These times were achieved by using the minimal levels of the filtering described above that gave any results at all. That is, in some of the spectra, the distribution of $\mathrm{S} / \mathrm{N}$ frequencies was such that a more relaxed value of the filter was required to have sufficient peaks to give any results. Typically, this involved starting with a value of 10 , as shown in Figure 1 , and the increasing the minimum frequency to get a clear result. As the minimum frequency was increased, the execution times increased, and at least part of the slower execution times in Table 2 are a result of this. The overall average execution time was $2.9 \mathrm{~s}$.

We were able to resolve the missing fragmentation 


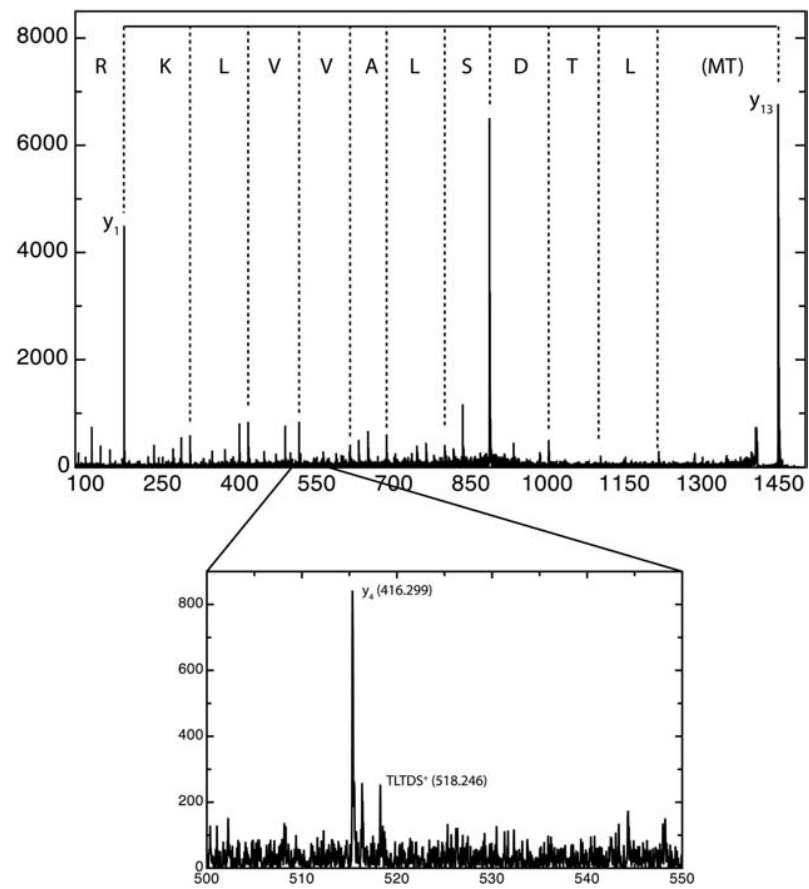

Figure 2. Fragmentation (MSMS) spectrum of b-galactosidase peptide MTLTDSLAVVLKR showing y-series ions. Inset shows the internal ion TLTDS $^{+}$that resolves the unfragmented $\mathrm{N}$ terminal sequence ambiguity.

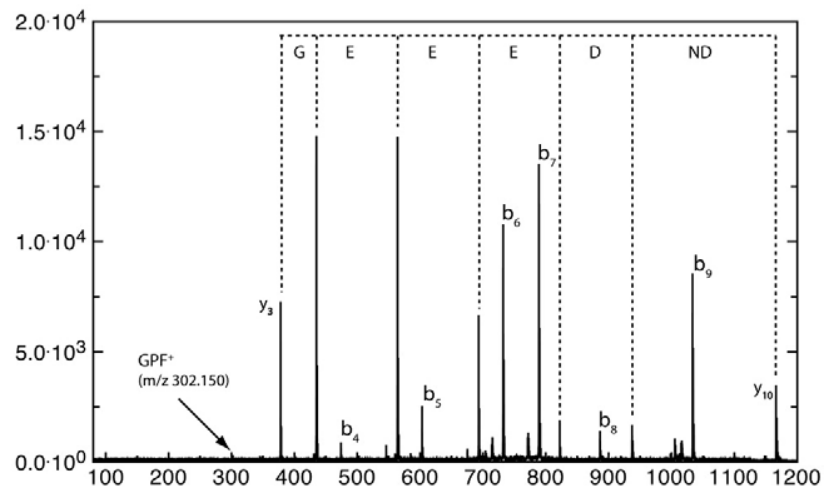

Figure 3. Fragmentation (MSMS) spectrum of synthetic peptide 2, DNDEEEGPFD showing an incomplete y-series, but a complete sequence due to the presence of complimentary b-series ions.

ambiguities for 11 of the 15 by locating internal fragments within the original spectra; at this stage of our development this was done manually. For example, the near isobaric missed fragment of b-galactosidase 954$962(\mathrm{~m} / \mathrm{z}$ 1083.522) was shown to have the sequence GDFQ.. by the presence of an internal fragment, $\mathrm{m} / \mathrm{z}$ 391.16 corresponding to . . .DFQ. . . t the N-terminal ambiguity of $\mathrm{m} / \mathrm{z} 1446.841 \mathrm{~b}$-galactosidase peptide was resolved by the internal fragment, $\mathrm{m} / \mathrm{z} 518.25$, corresponding to the sequence ... TXTDS. .., thereby placing the methionine residue at the amino terminus as expected, as illustrated in Figure 2. The C-terminal ambiguities of both the 402-412 peptide of BSA and angiotensin I were resolved in a similar fashion, i.e., the $\mathrm{m} / \mathrm{z}$ 285.14 ion corresponding to HF in the case of the BSA peptide and the two ions, $m / z 519.25$ and 632.33, corresponding to HPFH and XHPFH for angiotensin I. Note also that two of the three nontryptic peptides studied, angiotensin I and DNDEEEGPFD $\left(\mathrm{MH}^{+}=1166\right)$, were sequenced correctly. The annotated spectrum for the nontryptic DNDEEEGPFD is shown in Figure 3 and illustrates an extensive b-series that compensates for an incomplete set of $y$-series fragments. A similar process was followed for the incomplete fragmentations shown in Table 2 and was successful in all but three cases. The spectrum of the b-galactosidase peptide $232-238, \mathrm{~m} / \mathrm{z}$ 900.385 , had overall too low an intensity to identify any internal fragments. In the case of the remaining two peptides, b-galactosidase 168-189, $\mathrm{m} / \mathrm{z}$ 1394.732, and BSA 66-75, $m / z$ 1163.631, the internal fragments in the spectra did not yield unambiguous results.

As shown above, the $36.4 \mathrm{mDa}$ difference between lysine $(\mathrm{K})$ and glutamine $(\mathrm{Q})$ cannot be resolved unambiguously in every case. On the other hand, our results indicate that the use of independent compositional analysis for each peak tends to shift the sequence score in favor of the correct sequence, as shown by the unambiguous assignment of a $\mathrm{Q}$ or $\mathrm{K}$ residue in 13 of the 21 possible instances in this set of 20 peptides. This is also apparent from the correct assignment of the C-terminal lysine in the tryptic fragments. As our algorithm makes no assumptions about the origin of peptides, the possibility exists of obtaining an incorrect sequence ending in glutamine, but we do not find this. For example, in the BSA fragment 66-75 (XVNEXTEFAK), the C-terminus is assigned correctly and the incorrect sequence ending in $\mathrm{Q}$ is not a possibility because the mass accuracy excludes $Q$ from the compositional analysis for y9. An example of how a sequence is generated using LIPCUT peptide composition by residue mass for this specific example is shown in Table 3. Starting from the C-terminal, residue masses are added sequentially, but are shown in the alphabetical format used internally by our algorithm. The approach used in this work employs multiple comparisons of experimentally measured masses with the accurate theoretical masses in LIPCUT. It is these multiple determinations of mass that ultimately yields the best assignment of sequences; for the example shown in Table 3, the average error for use of $\mathrm{K}$ in the sequence is $16.2 \mathrm{mDa}$ while the average error for use of $\mathrm{Q}$ is 28 $\mathrm{mDa}$. The value of this approach is demonstrated more extensively in the occurrence of several glutamines, as in $\beta$-galactosidase fragments 963-974 and 623-631, which have triplicate tandem glutamines, the instance of $\mathrm{KIQ}$ degeneracy occurs only twice and once, respectively.

The overall process by which sequences are generated is shown in Table 4a. The large number of y-ion compositions within a $100 \mathrm{mDa}$ band centered on the measured $\mathrm{m} / \mathrm{z}$ demonstrates this algorithm's reliance on previous peaks for the generation of a few unique sequences; each rightward column-step represents one more restriction on the amino acid compositions as they 
Table 3. Peak masses and theoretical residual masses for the y-series of tryptic BSA fragment 66-75 (XVNEXTEFAK) shows the benefit of multiple independent error determinations in elucidating the $\mathrm{K} / \mathrm{Q}$ ambiguity even when correct determination is impossible with a $50 \mathrm{mDa}$ mass accuracy.

\begin{tabular}{|c|c|c|c|c|c|c|c|}
\hline $\mathrm{y}$ & Composition & $\begin{array}{l}\text { Measured } \\
\text { Peak }(\mathrm{m} / \mathrm{z})\end{array}$ & $\begin{array}{c}\text { Obs'd Residual } \\
\text { Mass }\end{array}$ & $\begin{array}{l}\text { Theoret Residual } \\
\text { Mass with } \mathrm{K}\end{array}$ & $\begin{array}{l}\text { Error with } \\
\mathrm{K}(\mathrm{mDa})\end{array}$ & $\begin{array}{c}\text { Theoret Residual } \\
\text { Mass with } \mathrm{Q}\end{array}$ & $\begin{array}{c}\text { Error with } \\
\mathrm{Q}(\mathrm{mDa})\end{array}$ \\
\hline 1 & $\mathrm{~K}$ & 147.110 & 128.092 & 128.095 & 3 & 128.059 & 43 \\
\hline 2 & AK & 218.152 & 199.134 & 199.132 & 2 & 199.096 & 38 \\
\hline 3 & AFK & 365.201 & 346.183 & 346.200 & 17 & 346.164 & 19 \\
\hline 4 & AEFK & 494.244 & 475.226 & 475.243 & 17 & 475.207 & 19 \\
\hline 5 & AEFKT & 595.292 & 576.274 & 576.291 & 17 & 576.254 & 20 \\
\hline 6 & AEFKTX & 708.379 & 689.361 & 689.375 & 14 & 689.338 & 23 \\
\hline 7 & AEEFKTX & 837.416 & 818.398 & 818.417 & 19 & 818.381 & 17 \\
\hline 8 & AEEFKNTX & 951.468 & 932.450 & 932.460 & 10 & 932.424 & 26 \\
\hline 9 & AEEFKNTVX & 1050.579 & 1031.561 & 1031.529 & 32 & 1031.492 & 69 \\
\hline 10 & AEEFKNTVXX & 1163.600 & 1144.582 & 1144.613 & 31 & 1144.576 & 6 \\
\hline
\end{tabular}

are queried for extensions of smaller compositions. The rightmost column for each row is always 1 because only 1 unique entry exists for any given combination.

While displaying a table for each inferior sequence possibility would prove useless, Table $4 \mathrm{~b}$ rendered with $\mathrm{Q}$ instead of $\mathrm{K}$, and shows how the algorithm differentiates between $\mathrm{K}$ and Q. At $m / z=1050.579$, no compositions exist that meet the criteria for extension of the AEEFNQTX to AEEFNQTVX (the addition of V). This branch progresses as a missed fragmentation, yielding a final sequence possibility of $X(\mathrm{VN}) \mathrm{EXTEFAQ}$, but the score is decreased due to the absence of the 1050.579 peak to support it.

A comparison of our results to those obtained using the only other software tailored to MALDI TOF/TOF spectra, the ABI GPS DeNovo Explorer software provided with the instrument, was undertaken in an attempt to validate our approach. The comparison demonstrated several advantages to the use of the approach presented here. The DeNovo Explorer generated correct sequences for only ten of the twenty-two peptides. More significantly however, when incorrect sequences were produced, they generally had substantial, more than three incorrect residues, regions of incorrect sequence. In the case of the b-gal 507-518, the DeNovo Explorer software derived a completely incorrect sequence. The origin of these incorrect results is unclear; manual validation of the fragmentation data could find no support for the incorrect sequences.

Several recent papers [17, 24-29] demonstrate charge derivatization methods for peptides before tandem mass spectrometric sequencing analysis. The derivatizations promote peptide fragmentation in the gas phase and/or simplify the spectrum by reducing the number of fragmentation pathways. Two notable examples [17, 29] also elucidate informatics methods to analyze the simplified fragment spectra. While charge derivatization may increase sensitivity, justification of the improvements on sequencing performance would be helpful. Introduction of more heuristics, such as the assumption that the modified peptide will fragment to generate only $y$ - or only b-ions, into the spectral sequencing diminishes com- putational complexity and so run time, but the justification for these heuristics remains dubious, for the spectral assignments often include supposedly forbidden ion types, and the mistaken assumption of ion type can lead to undesirable sequence shunting. It was shown in this paper that complete compositional analysis, which is made rapid with LIPCUT, is able to distinguish $y$ - and b-ions. Further, it was found that using the complementary ion types enriches sequence confidence and elucidates some ambiguities resulting from low mass accuracy.

\section{Conclusions and Future Directions}

The approach described here introduces a new basis for peptide de novo sequencing. With this approach, the peptide mass for which a complete and reliable de novo sequence can be obtained is extended beyond $\mathrm{m} / \mathrm{z} 1600$. In addition, the increased computational robustness afforded by the use of the LIPCUT dataset allows for the exploitation of composition enumeration within a mass accuracy consistent with unimolecular decompositions obtained in a MALDI tandem TOF instrument. Additional work will focus on two areas; (1) incorporation of what is now the manual process of evaluating internal ions, and (2) the use of MAARIAN with data obtained from other instruments, and ions formed by electrospray ionization. This will require modifications to consider multiple charge states in the spectrum. Of equal interest is the potential application of LIPCUT and MAARIAN to spectra involving electron capture dissociation (ECD).

We also envision an equally viable extension of MAARIAN to incorporate the ability to consider certain post-translational or chemical modifications. Preliminary efforts suggest that such changes will only subtly change the computational order and only modestly increase overall computation time for each modification considered. In addition to the LIPCUT database being available upon request, all of the peak lists used in this work are available in the Supplementary Material Section (which can be found in the electronic version of this 
Table 4. (a) Composition-based filtering of $\mathrm{m} / \mathrm{z} 1163.600$ using $\mathrm{K}$

\begin{tabular}{|c|c|c|c|c|c|c|c|c|c|c|c|}
\hline $\begin{array}{l}\text { Measured } \\
\mathrm{m} / \mathrm{z}\end{array}$ & $\begin{array}{c}\text { Total y- } \\
\text { compositions }\end{array}$ & $\begin{array}{l}\text { Subset } \\
\text { containing } \\
\mathrm{K}\end{array}$ & $\begin{array}{c}\text { Subset } \\
\text { containing } \\
\text { AK }\end{array}$ & $\begin{array}{l}\text { Subset } \\
\text { containing } \\
\text { AFK }\end{array}$ & $\begin{array}{l}\text { Subset } \\
\text { containing } \\
\text { AEFK }\end{array}$ & $\begin{array}{c}\text { Subset } \\
\text { containing } \\
\text { AEFKT }\end{array}$ & $\begin{array}{l}\text { Subset } \\
\text { containing } \\
\text { AEFKTX }\end{array}$ & $\begin{array}{l}\text { Subset } \\
\text { containing } \\
\text { AEEFKTX }\end{array}$ & $\begin{array}{c}\text { Subset } \\
\text { containing } \\
\text { AEEFKNTX }\end{array}$ & $\begin{array}{c}\text { Subset } \\
\text { containing } \\
\text { AEEFKNTVX }\end{array}$ & $\begin{array}{c}\text { Subset } \\
\text { containing } \\
\text { AEEFKNTVXX }\end{array}$ \\
\hline 147.110 & 3 & 1 & & & & & & & & & \\
\hline 218.152 & 3 & 1 & 1 & & & & & & & & \\
\hline 365.201 & 14 & 3 & 1 & 1 & & & & & & & \\
\hline 494.244 & 79 & 9 & 2 & 1 & 1 & & & & & & \\
\hline 595.292 & 274 & 43 & 7 & 3 & 1 & 1 & & & & & \\
\hline 708.379 & 688 & 227 & 81 & 21 & 2 & 1 & 1 & & & & \\
\hline 837.416 & 3377 & 1107 & 470 & 121 & 21 & 7 & 1 & 1 & & & \\
\hline 951.468 & 10707 & 3686 & 1701 & 416 & 102 & 33 & 8 & 2 & 1 & & \\
\hline 1050.579 & 11440 & 6017 & 3037 & 949 & 171 & 60 & 24 & 4 & 1 & 1 & \\
\hline 1163.600 & 55906 & 26255 & 14289 & 3681 & 911 & 243 & 88 & 14 & 2 & 1 & 1 \\
\hline \multicolumn{12}{|c|}{ (b) Composition-based filtering of m/z 1163.600 using Q } \\
\hline $\begin{array}{c}\text { Measured } \\
\mathrm{m} / \mathrm{z} \\
\end{array}$ & $\begin{array}{c}\text { Total y } \\
\text { compositions }\end{array}$ & $\begin{array}{c}\text { Subset } \\
\text { containing } \\
\mathrm{Q} \\
\end{array}$ & $\begin{array}{c}\text { Subset } \\
\text { containing } \\
\text { AQ } \\
\end{array}$ & $\begin{array}{c}\text { Subset } \\
\text { containing } \\
\text { AFO } \\
\end{array}$ & $\begin{array}{c}\text { Subset } \\
\text { containing } \\
\text { AEFO } \\
\end{array}$ & $\begin{array}{c}\text { Subset } \\
\text { containing } \\
\text { AEFOT } \\
\end{array}$ & $\begin{array}{l}\text { Subset } \\
\text { containing } \\
\text { AEFQTX } \\
\end{array}$ & $\begin{array}{c}\text { Subset } \\
\text { containing } \\
\text { AEEFOTX } \\
\end{array}$ & $\begin{array}{c}\text { Subset } \\
\text { containing } \\
\text { AEEFQNTX }\end{array}$ & $\begin{array}{c}\text { Subset } \\
\text { containing } \\
\text { AEEFQNTVX }\end{array}$ & $\begin{array}{c}\text { Subset } \\
\text { containing } \\
\text { AEEFQNTVXX } \\
\end{array}$ \\
\hline 147.110 & 3 & 1 & & & & & & & & & \\
\hline 218.152 & 3 & 1 & 1 & & & & & & & & \\
\hline 365.201 & 14 & 2 & 1 & 1 & & & & & & & \\
\hline 494.244 & 79 & 8 & 2 & 1 & 1 & & & & & & \\
\hline 595.292 & 274 & 30 & 5 & 2 & 1 & 1 & & & & & \\
\hline 708.379 & 688 & 124 & 48 & 16 & 1 & 1 & 1 & & & & \\
\hline 837.416 & 3377 & 810 & 354 & 113 & 16 & 7 & 1 & 1 & & & \\
\hline 951.468 & 10707 & 2906 & 1367 & 411 & 74 & 28 & 9 & 2 & 1 & & \\
\hline 1050.579 & 11440 & 2947 & 1447 & 434 & 61 & 19 & 9 & 0 & 0 & 0 & \\
\hline 1163.600 & 55906 & 17947 & 9709 & 2846 & 605 & 176 & 88 & 12 & 1 & 1 & 1 \\
\hline
\end{tabular}


article); all spectra will be made available as ASCII files upon request.

\section{Acknowledgments}

The authors thank Dr. Amina S. Woods of the Intramural Research Program, National Institute on Drug Abuse, for her generous gift the synthetic nontryptic peptides used in this work and Dr. Paul S. Blank for helpful discussions concerning signal processing. This work was supported by the Intramural Research Program of the National Institute of Child Health and Human Development, NIH.

\section{References}

1. Eng, J.; McCormack, A.; Yates, J. III. An Approach to Correlate Tandem Mass Spectral Data of Peptides with Amino Acid Sequences in a Protein Database. J. Am. Soc. Mass Spectrom. 1994, 5, 976-989.

2. Perkins, D. N.; Pappin, D. J.; Creasy, D. M.; Cottrell, J. S. ProbabilityBased Protein Identification by Searching Sequence Databases Using Mass Spectrometry Data. Electrophoresis 1999, 20, 3551-3567.

3. Geer, L. Y.; Markey, S. P.; Kowalak, J. A.; Wagner, L.; Xu, M.; Maynard, D. M.; Yang, X.; Shi, W.; Bryant, S. H. Open Mass Spectrometry Search Algorithm. J. Proteome Res. 2004, 3, 958-964.

4. Steen, H.; Mann, M. The ABC's (and XYZ's) of Peptide Sequencing. Nat. Rev. Mol. Cell. Biol. 2004, 5, 699-711.

5. Yergey, A. L.; Coorssen, J. R.; Backlund, P. S.; Blank, P. S.; Humphrey, G. A.; Zimmerberg, J.; Campbell, J. M.; Vestal, M. L. De Novo Sequencing of Peptides Using MALDI/TOF-TOF. J. Am. Soc. Mass Spectrom. 2002, 10, 784-791.

6. Koy, C.; Mikkat, S.; Raptakis, E.; Sutton, C.; Resch, M.; Tanaka, K.; Glocker, M. O Matrix-Assisted Laser. Desorption/Ionization-Quadrupole Ion Trap-Time of Flight Mass Spectrometry Sequencing Resolves Structures of Unidentified Peptides Obtained by In-Gel Tryptic Digestion of Haptoglobin Derivatives from Human Plasma Proteomes. Proteomics 2003, 3, 851-858

7. Yew, J. Y.; Dikler, S.; Stretton, A. O. De Novo Sequencing of Novel Neuropeptides Directly from Ascaris Sum Tissue Using MatrixAssisted Laser Desorption/Ionization Time-of-Flight/Time-of-Flight. Rapid Commun. Mass Spectrom. 2003, 17, 2693-2698.

8. Hanada, K.-I.; Yewdell, J. W.; Yang, J. C. Immune Recognition of a Human Renal Cancer Antigen Through Post-Translational Protein Splicing. Nature 2004, 427, 252-256.

9. Shui, W.; Liu, Y.; Bao, H.; Liang, S.; Yang, P.; Chen, X Enhancing TOF/TOF-based De Novo Sequencing Capability for High Throughput Protein Identification with Amino Acid-Coded Mass Tagging. J. Proteome Res. 2005, 4(1), 83-90.

10. Dancik, V.; Addona, T. A.; Clauser, K. R.; Vath, J. E.; Pevzner, P. A. De Novo Peptide Sequencing via Tandem Mass Spectrometry. J. Comput. Biol. 1999, 6(3/4), 327-342.

11. Zhang, Z.; McElavin, J. S. De Novo Peptide Sequencing by TwoDimensional Fragment Correlation Mass Spectrometry. Anal. Chem. 2000, 72, 2337-2350.
12. Chen, T.; Kao, M.-Y.; Tepel, M.; Rush, J.; Church, G. A Dynamic Programming Approach to de Novo Peptide Sequencing via Tandem Mass Spectrometry. J. Comput. Biol. 2001, 8(3), 325-337.

13. Taylor, J. A.; Johnson, R. S. Implementation and Uses of Automated de Novo Peptide Sequencing by Tandem Mass Spectrometry. Anal. Chem. 2001, 73, 2594-2604.

14. Ma, B.; Zhang, K.; Hendrie, C.; Liang, C.; Li, M.; Doherty-Kirby, A.; Lajoie, G. PEAKS: Powerful Software for Peptide de Novo Sequencing by Tandem Mass Spectrometry. Rapid Commun. Mass Spectrom. 2003, 17, 2337-2342.

15. Spengler, B. De Novo Sequencing, Peptide Composition Analysis, and Composition-Based Sequencing: A New Strategy Employing Accurate Mass Determination by Fourier Transform Ion Cyclotron Resonance Mass Spectrometry. J. Am. Soc. Mass Spectrom. 2004, 15, 703-714.

16. Zhang, Z. De Novo Peptide Sequencing Based on a Divide and Conquer Algorithm and Peptide Tandem Spectrum Simulation. Anal. Chem. 2004, 76, 6374-6383.

17. Raucci, G.; Gabrielli, M.; Novelli, S.; Picariello, G.; Collins, S. H. CHASE, a Charge-Assisted Sequencing Algorithm for Automated HomologyBased Protein Identifications with Matrix-Assisted Laser Desorption/ Ionization Time-of-Flight Post-Source Decay Fragmentation Data. J. Mass Spectrom. 2005, 40, 475-488.

18. PRG2005 De Novo Peptide Sequencing Results. http://www.abrf.org/ index.cfm/group.show/Proteomics.34.htm\#R_2; 2005.

19. Kapp, E. A.; Schutz, F.; Reid, G. E.; Eddes, J. S.; Moritz, R. L.; O'Hair, R. A.; Speed, T. P.; Simpson, R. J. Mining a Tandem Mass Spectrometry Database to Determine the Trends and Global Factors Influencing Peptide Fragmentation. Anal. Chem. 2003, 75, 6251-6264.

20. Medzihradszky, K.; Campbell, J.; Baldwin, M.; Juhasz, P.; Vestal, M.; Burlingame, A. The Characteristics of peptide Collision-Induced Dissociation Using a High-Performance MALDI-TOF/TOF Tandem Mass Spectrometer. Anal. Chem. 2000, 72, 552-558.

21. Olson, M.; Bostic, K.; Seltzer, M. Proceedings of the Summer Usenix Technical Conference; Monterey, CA, June, 1999.

22. http://biowulf.nih.gov

23. Epstein, J. A.; Olson, M. T.; Yergey, A. L., unpublished.

24. Spengler, B.; Lutzenkirchen, F.; Metzger, S.; Chaurand, P.; Kaufmann, R.; Jeffery, W.; Bartlet-Jones, M.; Pappin, D. Peptide Sequencing of Charged Derivatives by Postsource Decay MALDI Mass Spectrometry. Int. J. Mass Spectrom. Ion Porcesses 1997, 169/170, 127-140.

25. Adamczyk, M.; Gebler, J. C.; Wu, J. Charge Derivitization of Peptides to Simplify Their Sequencing with an Ion Trap Mass Spectrometer. Rapid Commun. Mass Spectrom. 1999, 13, 1413-1422.

26. Keough, T.; Lacey, M.; Youngquist, R. Solid-Phase Derivatization of Tryptic Peptides for Rapid Protein Identification by Matrix-Assisted Laser Desorption/Ionization Mass Spectrometry. Rapid Commun. Mass Spectrom. 2002, 16, 1003-1015.

27. Marekov, L. N.; Steinert, P. M. Charge Derivatization by 4-Sulfophenyl Isothiocyanate Enhances Peptide Sequencing by Post-Source Decay Matrix-Assisted Laser Desorption/Ionization Time-of-Flight Mass Spectrometry. J. Mass Spectrom. 2003, 38, 373-377.

28. Booth, S. A.; Hohnholt, S. G.; Papayannopoulos, I. A. Sequence Determination of Glutathione S-Transferase from Horse by MALDI Tandem Time-ofFlight Mass Spectrometry; 52nd ASMS Conference on Mass Spectrometry and Allied Topics; Nashville, TN, May, 2004.

29. Differential Mapping of Serum Fractions Using iTRAQ TM Reagents and LC-MS/MS on the 4800 MALDI TOF/TOF TM Analyzer. http:// www.appliedbiosystems.com; 2005. 